\title{
THE EFFECTS OF MAGNETIC FIELD MORPHOLOGY ON THE DETERMINATION OF OXYGEN AND IRON ABUNDANCES IN THE SOLAR PHOTOSPHERE
}

\author{
Christopher S. Moore ${ }^{1}$, Han Uitenbroek ${ }^{2}$, Matthias Rempel $^{3}$, Serena Criscuoli $^{2}$, and Mark P. Rast ${ }^{4}$ \\ ${ }^{1}$ Department of Astrophysical and Planetary Sciences, Center for Astrophysics and Space Astronomy, \\ University of Colorado, Boulder, CO 80309, USA \\ ${ }^{2}$ National Solar Observatory, Sunspot, NM 88349, USA \\ ${ }^{3}$ High Altitude Observatory, National Center for Atmospheric Research, Boulder, CO 80307, USA \\ ${ }^{4}$ Department of Astrophysical and Planetary Sciences, Laboratory for Atmospheric and Space Physics, \\ University of Colorado, Boulder, CO 80309, USA; mark.rast@lasp.colorado.edu \\ Received 2014 August 11; accepted 2014 November 21; published 2015 January 23
}

\begin{abstract}
We have explored the impact of magnetic fields on the determination of the solar photospheric oxygen and iron abundances using three-dimensional radiation-magnetohydrodynamic (MHD) simulations of convection. Specifically, we examined differences in abundance deduced from three classes of atmospheres simulated with the MURaM code: a pure hydrodynamic (HD) simulation, an MHD simulation with a local dynamo magnetic field that has saturated with an unsigned vertical field strength of $80 \mathrm{G}$ at $\tau=1$, and an MHD simulation with an initially imposed vertical mean field of $80 \mathrm{G}$. We use differential equivalent width analysis for diagnosing abundances derived from five oxygen and four iron lines of differing wavelength, oscillator strength, excitation potential, and Landé $g$-factor, and find that the morphology of the magnetic field is important to the outcome of abundance determinations. The largest deduced abundance differences are found in the vertical mean field simulations, where the $\mathrm{O}$ I and $\mathrm{Fe}$ I abundance corrections compared to the pure HD case are $\sim+0.011$ dex and +0.065 dex respectively. Small scale unresolved field resulting from the local dynamo has a smaller impact on abundance determinations, with corrections of -0.0001 dex and +0.0044 dex in the magnetized compared to the pure HD simulations. While the overall influence of magnetic field on abundance estimates is found to be small, we stress that such estimates are sensitive not only to the magnitude of magnetic field but also to its morphology.
\end{abstract}

Key words: Sun: abundances - Sun: granulation - Sun: magnetic fields - Sun: photosphere

\section{INTRODUCTION}

Recent downward revisions to the solar abundance of carbon, nitrogen, and oxygen resulted from the implementation of full three-dimensional (3D) convective simulations in the modeling effort (Asplund et al. 2009). Specifically, the change in the oxygen abundance has been dramatic, with a decrease from $A_{\mathrm{O}}=8.83^{5}$ (Grevesse \& Sauval 1998) to $A_{\mathrm{O}}=8.66$ (Asplund et al. 2004, 2005; Grevesse et al. 2007), and then further revision to the most recent value of $A_{\mathrm{O}}=8.69$ (Asplund et al. 2009) with the inclusion of line blends and revised weighted oscillator strength, $g f$, values (Allende Prieto et al. 2001; Asplund et al. 2004; Nordlund et al. 2009). While the newer oxygen abundance somewhat alleviates the disparity between the larger solar value and that derived from local B stars (Meyer et al. 1998; Nissen et al. 2002), it is quite different from the value consistent with helioseismological measurements (Christensen-Dalsgaard et al. 2009; Nordlund et al. 2009; Asplund et al. 2009), which is much closer to the solar value originally derived from one-dimensional (1D) modeling.

Since even the "quiet" Sun is likely magnetically structured at sub-granular scales (see Martínez Pillet 2013 for a recent review), a relevant and open question remains how sensitive abundance determinations are to the presence and structure of magnetic fields. Borrero (2008) explored abundance sensitivity in 1D magnetic model atmospheres and found that the derived abundances can suffer from a systematic error of up to 0.1 dex if fields are ignored. Fabbian et al. (2010) and Fabbian et al.

\footnotetext{
5 Typically abundances are quoted as $\log _{10}\left(\epsilon_{X} / \epsilon_{H}\right)=A_{X}-12$, where $\epsilon_{X}$ is the absolute abundance of the element $X, \epsilon_{H}$ is the absolute abundance of hydrogen, and $A_{X}$ is the element $X$ abundance in dex.
}

(2012) analyzed iron abundances in 3D magnetohydrodynamic (MHD) simulations with imposed vertical mean magnetic fields and deduced that the values can differ by $\sim 0.03-0.11$ dex from the values found in purely hydrodynamic (HD) solutions. We supplement their analysis here by investigating the sensitivity of iron and oxygen abundance determinations to fields generated by the action of a local dynamo (LD). A local dynamo field has a significantly different topology than one obtained from mean field simulations, and thus has a subtly different effect on abundance determinations, even when the unsigned flux is of comparable magnitude.

To determine the abundance error made if such fields exist and are not accounted for, we compared the equivalent widths of four iron and five oxygen lines calculated from the three classes of (M)HD simulations, using the HD simulation as a reference. The assumption we make is that observed line profiles are compared with line profiles computed from pure HD simulations, as has been the prevailing practice until recently. If the spectra from the real Sun are significantly modified by the presence of small scale magnetic field, there is an error in the abundance determination. Thus, following the procedure adopted by Fabbian et al. (2010), we define the abundance correction as the difference between the abundance employed in the synthesis and the one derived comparing the equivalent width from the MHD simulations with the curves of growth obtained from the HD snapshots. For example, if the presence of the magnetic field reduces the equivalent width of a spectral line of a given element compared to the non-magnetic case, then by comparison with the curves of growth we would deduce a smaller abundance, so that the correction would be positive.

Like Fabbian et al. (2010, 2012), we find that in solutions computed with an imposed mean field, the dominant cause for 


\begin{tabular}{lccccccccc}
\multicolumn{10}{c}{ Table 1 } \\
O I Lines
\end{tabular}

Notes. The five oxygen lines used in this analysis (rest wavelengths quoted in air). Also displayed are the oxygen abundance and equivalent width results. \langle\rangle is the arithmetic mean of the five lines. The uncertainties, $\sigma_{\Delta A}$, are derived from the temporal standard deviation of equivalent widths over 10 snapshots in each magnetic case.

${ }^{\text {a }}$ Designates a forbidden line

changes in the equivalent width, and hence in abundance estimates, is the thermodynamic change induced by the presence of organized magnetic field. Strong and dense magnetic concentrations commonly found in intergranular lanes decrease the local density and opacity, allowing deeper, hotter layers to be exposed on equal optical depth surfaces (Schuessler \& Solanki 1988; Vögler 2005). In these imposed field solutions, the implied abundance corrections reflect differences in the observed temperature structure, with the direct Zeeman broadening influence of the magnetic field of secondary importance at visible wavelengths (Fabbian et al. 2010; Criscuoli et al. 2013). In simulations where the field is generated by the local dynamo alone, induced thermodynamic changes are smaller and the abundance corrections are determined by a combination of the effects of these and direct Zeeman broadening, depending on the properties of the line, its formation height, Landé factor, and excitation potential.

Our paper is organized as follows. In Section 2 we briefly discuss the atmospheric model and in Section 3 the spectral synthesis code. In Section 4 we discuss abundance results and their underlying causes and in Section 5 we provide conclusions.

\section{SIMULATED ATMOSPHERES}

The simulations used in this investigation employ the Max-Planck-Institute for Solar System Research/University of Chicago Radiation Magneto-hydrodynamics code (Vögler et al. 2005; Rempel et al. 2009; Rempel 2014). The code was used to solve the non-ideal MHD equations on a domain of $6.144 \times 6.144 \times 3.072 \mathrm{Mm}^{3}$, with $768 \times 768 \times 384$ grid points and a uniform grid spacing of $8 \mathrm{~km}$, the minimum spacing needed to properly describe the spatial spectrum of energy distribution (Rempel 2014).

We employed the output of three simulations as input atmospheres for spectral synthesis and abundance analysis. The first were obtained from a pure HD run. The second are from a MHD run with 0 net vertical flux $\left(\left\langle B_{z}\right\rangle=0 \mathrm{G}\right)$, capturing the atmospheric properties of a ubiquitous local dynamo (LD, hereafter). The LD simulation was initialized by adding a randomly oriented seed field of $\|\mathbf{B}\|=10^{-3} \mathrm{G}$ to the thermally relaxed HD run. The solution was then evolved until the magnetic field amplitude saturated. The field saturation amplitude in the photosphere of the LD solution depends on the boundary condition employed at depth, with the solution used in this study saturating at an unsigned vertical flux density of approximately $80 \mathrm{G}$ at $\tau=1$ (see Rempel 2014 for more details). To test whether the manner in which the field is introduced into the simulations influences the thermodynamic structure of the atmosphere in a way that consequently impacts abundance determinations, we also generated atmospheres with mean vertical magnetic field (MF solution, hereafter). A vertical mean field of $80 \mathrm{G}$ was introduced into the relaxed HD solution, and the simulation was evolved to a statistically steady state. We note that, because of local dynamo effects, the unsigned vertical field in the MF simulation saturated at approximately $120 \mathrm{G}$. The two MHD cases thus represent different magnetic morphologies with comparable, though not equal, net unsigned flux densities (about $80 \mathrm{G}$ for the LD case and about $120 \mathrm{G}$ for the MF case).

Figure 1 displays the magnetic field distribution on the $630 \mathrm{~nm}$ optical depth unity surface in snapshots of each of the two magnetized simulations. Note the differences in the field distribution. The MF simulation (right panels) displays more organized vertical field concentrated within intergranular lanes. The LD simulation is dominated by more pervasive moderate strength magnetic field. Note also the depressed optical depth unity height at the locations of strong, concentrated vertical field in the bottom panels. These are the regions most responsible for the changes in the thermodynamic properties on $\tau$ surfaces that yield smaller equivalent widths and positive abundance corrections. In addition, these are sites of large "radiation leaks" that cause the average thermal gradient with height to be shallower in the MF simulation than in the LD simulation (cf. Fabbian et al. 2010; Criscuoli \& Uitenbroek 2014), contributing to a stronger reduction in equivalent width in the former than in the latter.

\section{SPECTRAL SYNTHESIS}

We performed spectral synthesis using 10 snapshots of each of the 3 simulated atmospheres separated by 4 minute intervals. The spectral synthesis was conducted using the RH code (Uitenbroek 2001) for vertical lines of sight $(\mu=1)$. For computational expediency, we assumed Local Thermodynamic Equilibrium (LTE) and we synthesized spectra at every fourth horizontal data point in each direction. We checked that this downsampling had negligible effect on the spatially averaged spectral line shapes and equivalent widths.

The spectral lines chosen for this study are those of $\mathrm{O}$ I and $\mathrm{Fe}$ I in common with those used by Asplund et al. (2004) and Fabbian et al. (2012), respectively. The lines are listed in Tables 1 and 2 and span different wavelength, excitation potential, oscillator strength, and Landé factors. It is important 

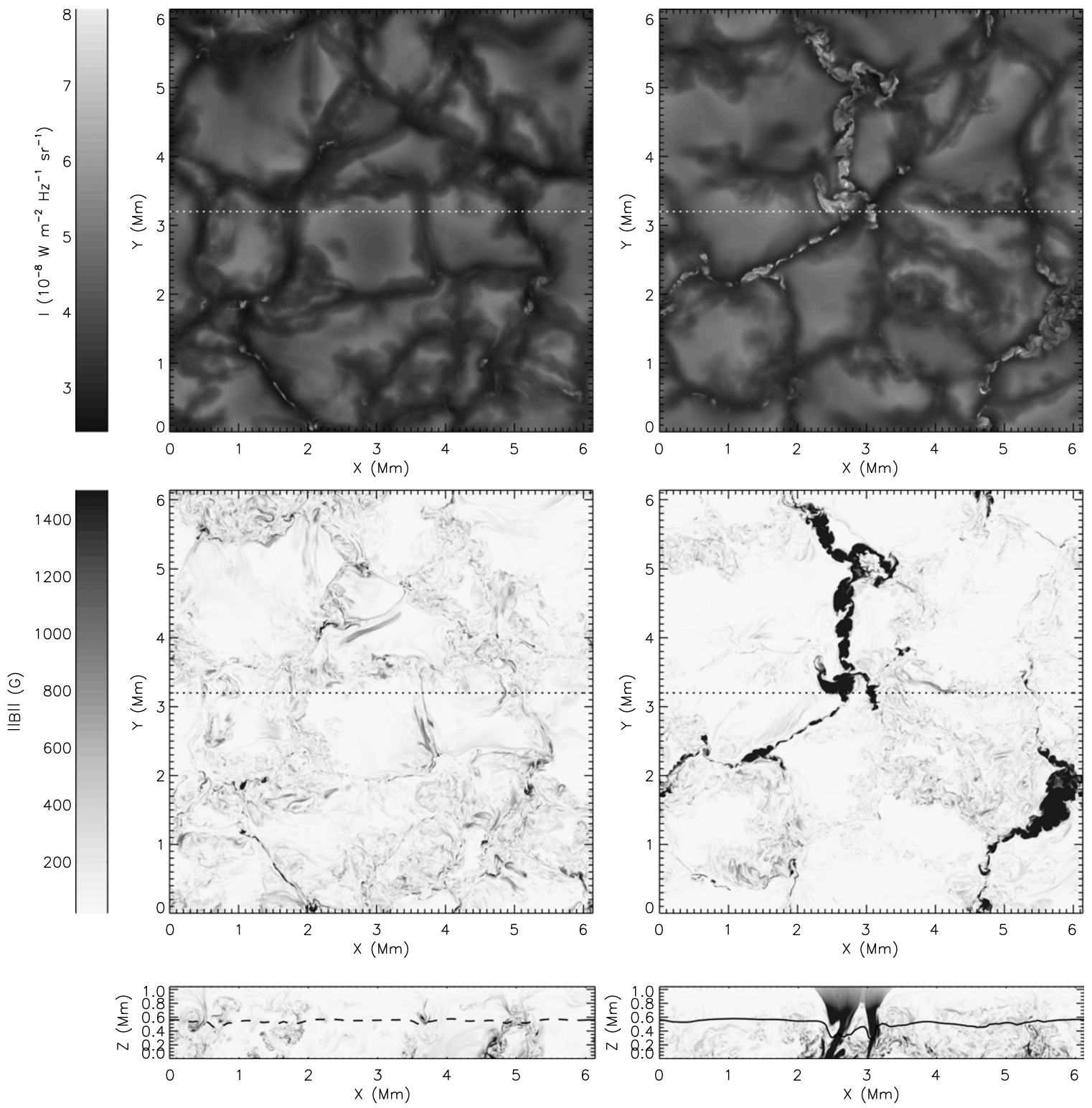

Figure 1. Top panel: Snapshots of the emergent continuum intensity images at $630 \mathrm{~nm}$ in the local dynamo (LD, left) and mean field (MF, right) simulations. Note the higher intensity of the magnetic features in the intergranular lanes. Middle row: Images of the magnetic field magnitude in the LD (left) and MF (right) atmospheres on the optical depth unity surface. The horizontal dotted line in the top and middle panels indicate the horizontal position of the vertical magnetic magnitude slice in the bottom panel. Bottom row: A vertical slice of the magnetic field magnitude for the LD (left) and MF (right) atmospheres. The dashed and solid curves indicate the optical depth unity heights in the LD and MF simulation snapshots respectively. To demonstrate the structural differences in the atmospheres, display thresholds were set at $20 \mathrm{G}$ and $1.5 \mathrm{kG}$ for both simulations.

Table 2

Fe I Lines

\begin{tabular}{|c|c|c|c|c|c|c|c|c|c|}
\hline $\begin{array}{l}\lambda \\
{[\mathrm{nm}]}\end{array}$ & Term & $\log g f$ & $\begin{array}{l}\chi_{\mathrm{exc}} \\
{[\mathrm{eV}]}\end{array}$ & $g_{L}$ & $\begin{array}{l}W_{\mathrm{HD}} \\
{[\mathrm{pm}]}\end{array}$ & $\begin{array}{c}\Delta A_{\mathrm{Fe}} \\
{[\mathrm{dex}]} \\
{[\mathrm{HD}-\mathrm{LD}]}\end{array}$ & $\begin{array}{c}\sigma_{\Delta A_{\mathrm{Fe}}} \\
{[\mathrm{dex}]} \\
{[\mathrm{HD}-\mathrm{LD}]}\end{array}$ & $\begin{array}{c}\Delta A_{\mathrm{Fe}} \\
{[\mathrm{dex}]} \\
{[\mathrm{HD}-\mathrm{MF}]}\end{array}$ & $\begin{array}{c}\sigma_{\Delta A_{\mathrm{Fe}}} \\
{[\mathrm{dex}]} \\
{[\mathrm{HD}-\mathrm{MF}]}\end{array}$ \\
\hline $524.705^{\mathrm{a}}$ & $a^{5} \mathrm{P}-z^{7} \mathrm{D}$ & -4.946 & 0.0873 & 2.00 & 6.8334 & +0.0123 & 0.0292 & +0.0825 & 0.0278 \\
\hline $525.021^{\mathrm{a}}$ & $a^{5} \mathrm{D}-z^{7} \mathrm{D}$ & -4.938 & 0.12 & 3.00 & 6.7274 & +0.0048 & 00295 & $\begin{array}{r}+0.0784 \\
+0.0784\end{array}$ & 0.0274 \\
\hline 525.065 & $a^{5} \mathrm{P}-y^{5} \mathrm{P}$ & -2.050 & 2.19 & 1.50 & 10.0918 & +0.0071 & 0.0233 & +0.0463 & 0.0205 \\
\hline 630.249 & $e^{5} \mathrm{D}-z^{5} \mathrm{P}$ & -1.131 & 3.686 & 2.50 & 8.1883 & -0.0065 & 0.0241 & +0.0516 & 0.0199 \\
\hline$\overline{\langle\rangle}$ & & & & & & +0.0044 & & +0.065 & \\
\hline
\end{tabular}

Notes. The five iron lines used in this analysis (rest wavelengths quoted in air). Also displayed are the iron abundance and equivalent width results. \langle\rangle is the arithmetic mean of the five lines. The uncertainties, $\sigma_{\Delta A}$, are derived from the temporal standard deviation of equivalent widths over 10 snapshots in each magnetic case.

${ }^{a}$ Designates a forbidden line 


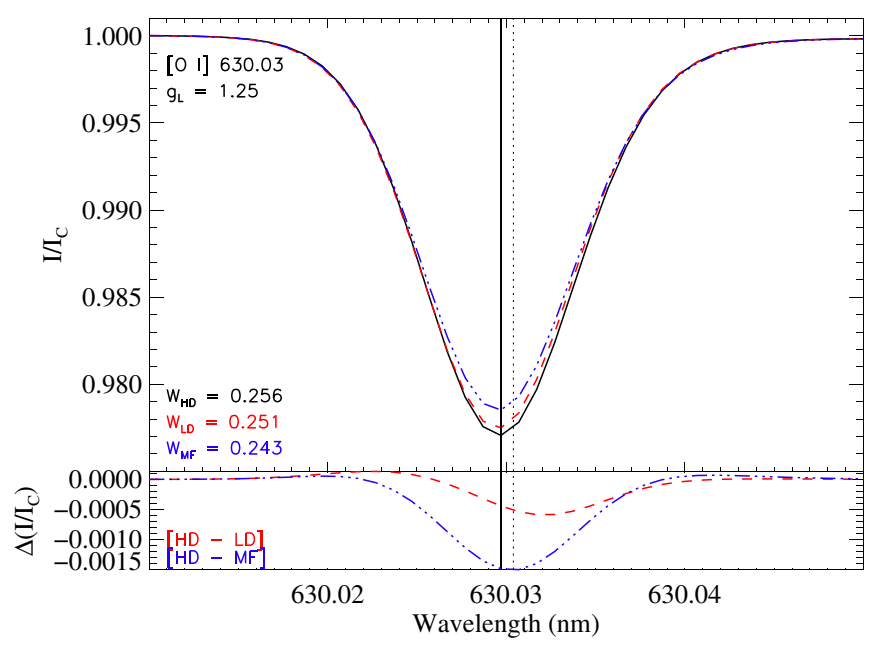

Figure 2. Temporally and spatially averaged [O $\mathrm{I}] 630.030 \mathrm{~nm}$ line profile in the hydrodynamic (HD), local dynamo (LD), and mean field (MF) simulation atmospheres, with differences relative to the HD case shown in the bottom panel. Top panel: the black solid line is the profile deduced from the HD simulation, the red dashed line is that determined using the LD solution, and the blue dashdotted line is the spectral line profile from the MF atmosphere. The black solid vertical line in the bottom panel is the HD solution line core wavelength and the black dotted vertical line is the rest wavelength. Spectral lines synthesized using the magnetized simulations are shallower than those using the HD atmosphere. The reduction in depth is three times larger for the MF solution than the LD due to the strong indirect influence of the field on the atmospheric temperature stratification in those simulations.

to note that the chosen lines have been widely investigated, especially in the context of solar abundance determination. In particular, numerous studies have pointed out the necessity of detailed non-LTE computation of the O I 777 triplet for a correct interpretation of the observations and derivation of oxygen abundance (e.g., Kiselman 1991; Asplund et al. 2004; Allende Prieto et al. 2004; Pereira et al. 2009; Fabbian et al. 2009). The purpose of this study is to estimate the effects of the magnetic field on the determination of abundances by comparison of spectra synthesized using HD versus MHD simulation solutions. We do not make direct comparisons with observations, for which full non-LTE synthesis would be required. The inclusion of nonLTE effects would quantitatively affect our results, but would not alter the general conclusions.

We performed forward spectral synthesis of lines using the HD simulation atmosphere and abundances of $-0.10,-0.05$, -0.02 , and +0.03 dex, relative to a nominal value. The nominal spectral synthesis oxygen abundance value was $A_{\mathrm{O}}=8.69 \mathrm{dex}$ and iron was $A_{\mathrm{Fe}}=7.44 \mathrm{dex}$. With the resulting $\mathrm{HD}$ equivalent widths we created curves of growth (see, e.g., Gray 2005). The curves of growth were linear over the abundance ranges examined, and were used to estimate abundances from line profiles synthesized with the LD and MF atmospheres and nominal abundance values. This is consistent with the goal of estimating the error introduced into observational assessments of solar abundances by a magnetic field that is either not known to exist or left out of the spectral analysis.

\section{ABUNDANCE RESULTS AND THE INFLUENCE OF MAGNETIC MORPHOLOGY}

A very important factor in spectral line equivalent width changes, and consequent abundance determinations is the local change in thermodynamic stratification induced by strong field concentrations. The additional pressure supplied by strong $(|\mathbf{B}| \sim 1 \mathrm{kG})$ concentrations of field in intergranular lanes re-

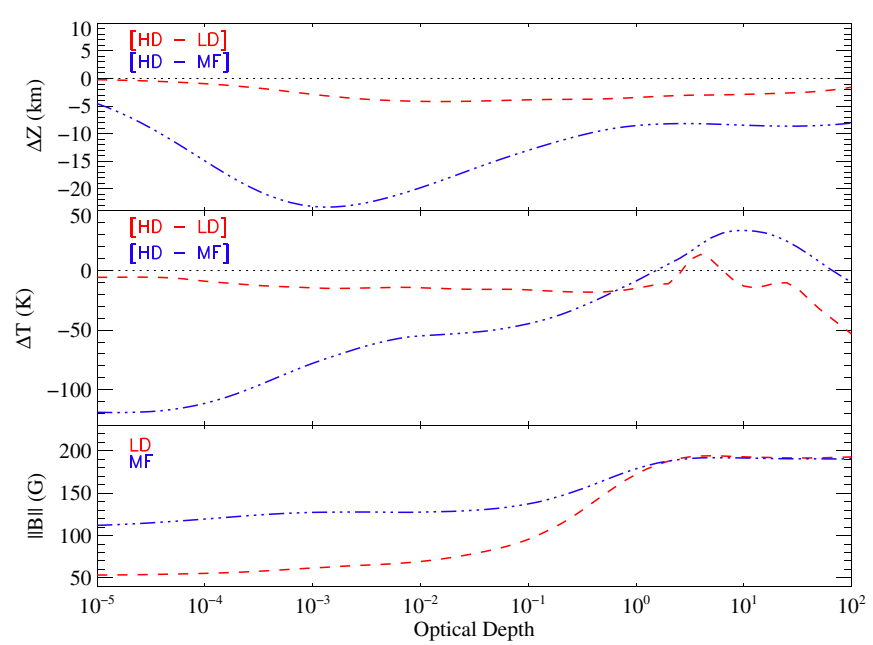

Figure 3. Differences between the thermodynamic properties of the hydrodynamic (HD) atmosphere and the magnetized atmospheres as a function of optical depth. Red dashed curves plot differences between the HD local dynamo (LD) simulations, while blue dash-dotted curves plot those between the $\mathrm{HD}$ and mean field (MF) solutions. Top panel: the differences in the average geometrical height with optical depth. Middle panel: temperature differences. Bottom panel: differences in the average magnitude of the magnetic field. The differences in all cases are larger in the MF than the LD simulation.

duces the particle density and thus decreases the local opacity. The lower opacity allows photons to escape from deeper layers in the evacuated light conduits and thus one observes higher temperatures. This causes an increase in the line source function at each optical depth, yielding less deep lines, particularly in the line core. Figure 2 illustrates the decrement in the line depth of the forbidden [O I] $630.030 \mathrm{~nm}$ line. It is consistent with results for iron lines in the study by Fabbian et al. (2012). The less deep spectral lines have smaller equivalent widths, thus a smaller abundance is deduced if a non-magnetic atmosphere is employed to interpret the data. As explained in Section 1, we call this a positive abundance correction. Figure 2 also shows that, as a consequence of the suppression of the velocity fields due to the presence of magnetic field concentrations (Criscuoli 2013), the line obtained from the MF simulations appears more symmetric than do the lines obtained from the HD and LD simulations.

In contrast to the indirect thermodynamic influence of the magnetic field on the line width, the direct magnetic influence on the line, the Zeeman splitting, primarily acts to increase the lines equivalent width. The magnitude of the Zeeman broadening varies depending on the line's Landé factor, and the magnetic influences, working in opposite directions, cancel to varying degree depending on the atomic and spectral line parameters and the atmospheric properties. Plots in Figure 3 show the difference between the HD and magnetized atmospheres' average optical depth unity height and temperature profiles, together with the height variation of the average magnetic field in the MHD simulations. The temperature differences between the HD and LD snapshots are small and thus so too are the indirect effects of the magnetic field on abundance determinations.

By contrast, the indirect thermodynamic influence of the magnetic field is more important in the MF simulation. Averaged over optical depth surfaces, the HD and MF solutions show a maximum temperature difference of almost $120 \mathrm{~K}$. This results from the large concentrations of relatively stronger field in the intergranular lanes $(\|\mathbf{B}\|>1 \mathrm{kG})$, which are characterized by a shallower temperature gradient. The consequent MF spectra 

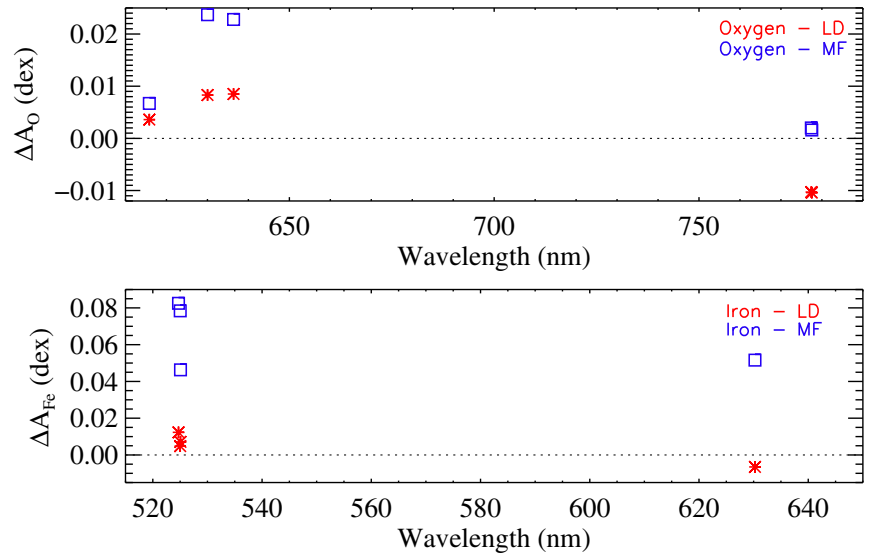

Figure 4. Summary of abundance results for O I and Fe I vs. wavelength. Top panel: The O I line results. Bottom panel: The Fe I lines. The asterisks are the local dynamo (LD) and the squares are the mean field (MF) results.

show shallower line depths when compared to the LD and HD spectra (Figure 2) due to the increased source function at the line formation height. We note that we compute the spectra from the resolved MHD solutions, not based on the mean profiles shown for illustration only in Figure 3. This is essential, as the average spectrum of the atmosphere is not equivalent to the spectrum of the average atmosphere (Uitenbroek \& Criscuoli 2011).

The cumulative effect of the magnetic fields of moderate strength that cover large areas in the LD simulation is to increase the relative importance of Zeeman broadening. In that simulation, fields with strengths between $25 \mathrm{G}<|\mathbf{B}|<1 \mathrm{kG}$ cover $\sim 90 \%$ of the surface at $\tau=1$. By contrast, only $(\sim 65 \%)$ of the $\tau=1$ surface is covered by moderate strength magnetic field in the MF simulation, and the integrated contribution of the Zeeman effect is not as large. The combined Zeeman contribution over the domain in the LD atmospheres is comparable in magnitude to the indirect thermodynamic contribution, and, since acting in opposite direction, can be sufficient to increase the equivalent width of some of the visible lines analyzed. Since the magnitude of the direct Zeeman broadening depends on the line's Landé factor, the resulting sign of the abundance correction depends on the underlying atomic properties of the line. By contrast, in the MF atmosphere the change in thermodynamic stratification is always the most important factor at visible wavelengths, and the abundance correction is always positive.

The results of our investigation are summarized in Tables 1 and 2 for the oxygen and iron lines, respectively. The tables report relevant line atomic parameters, the average equivalent width computed from the HD snapshots $\left(W_{\mathrm{HD}}\right)$, and the average $(\Delta A)$ and standard deviations $\left(\sigma_{\Delta A}\right)$ of the abundance corrections computed over the LD and MF snapshots. In general the influence of magnetic field on the abundances of oxygen and iron are small. In both magnetized atmospheres the abundance changes introduced by the field are much smaller than the ones required by the change from $1 \mathrm{D}$ to $3 \mathrm{D}$ atmospheric modeling.

\subsection{Oxygen Abundance Estimates}

The abundance corrections obtained for the oxygen lines are summarized in Table 1 and in the top panel of Figure 4 . The largest abundance correction is about $+5.6 \%(\sim 0.024 \mathrm{dex})$ and was obtained using the well studied forbidden $630.030 \mathrm{~nm}$ line and the MF atmosphere. This line is likely a reliable diagnostic for abundance(Asplund et al. 2004; Ayres 2008). For the reasons explained above, all oxygen line estimates derived using the MF atmosphere yield positive abundance corrections.

For the LD atmosphere, the sign of the abundance correction is largely determined by the formation height of the lines and their sensitivity to the magnetic field. In general, lines with higher excitation potential form deeper in the atmosphere, where the magnetic field is also stronger (see Figure 3). The effect is enhanced by the fact that in atmospheres altered by the presence of the magnetic field the line formation shifts to even deeper layers. The O 777 lines form deeper than the O I 630.030 and $636.377 \mathrm{~nm}$ lines (Allende Prieto et al. 2004), and due to their higher Landé factor are more sensitive to the magnetic field. This lead to negative abundance corrections. The O I $615.818 \mathrm{~nm}$ line also forms very deep in the atmosphere. The slight positive abundance correction found for this line is due to the relatively small Landé factor of the transition, which reduces the importance of Zeeman broadening.

Overall, the OI derived abundance errors introduced by magnetic fields in these lines is small (no larger than a few percent).

\subsection{Iron Abundance Estimates}

Table 2 and bottom panel of Figure 4 summarize the results obtained for the $\mathrm{Fe}_{\mathrm{I}}$ lines. The largest positive magnetic field induced abundance corrections obtained by our analysis of these lines are for the MF case, with an arithmetic mean in visible wavelength lines yielding $\sim+16 \%(\sim+0.065 \mathrm{dex})$. The line with the largest upward revision, the $524.705 \mathrm{~nm}$ line, shows a change of $+21 \%(\sim+0.083 \mathrm{dex})$. This is consistent with results reported by Fabbian et al. (2012), who found a change of +0.09 dex in models with a $100 \mathrm{G}$ imposed vertical mean field (which is their closest value to our $80 \mathrm{G}$ vertical mean field simulation).

Iron lines yield a similar distribution of positive and negative abundances corrections in the LD atmosphere as the oxygen lines. The $630.249 \mathrm{~nm}$ line, the one with the highest excitation potential, yields a negative correction of $\sim-1.5 \%$, as it forms deeper in the atmosphere, especially in the presence of magnetic field concentrations. All three $500 \mathrm{~nm}$ range Fe I lines computed for the LD give consistent positive corrections (up to $\sim+2.8 \%$ ) and form in the upper layers of the atmosphere (see for instance Khomenko \& Collados 2007 for a comparison of formation heights of the $630.2,524.7$, and $525.0 \mathrm{~nm}$ lines). As seen for $\mathrm{O}$ I, the local dynamo fields yield only small Fe I abundance corrections.

\section{CONCLUSION}

The solar photosphere is likely filled with magnetic field even in "quiet" Sun regions on granular and sub-granular size scales. These alter the velocity structure, thermodynamic properties, and the emergent radiation field depending on the field morphology (Schuessler \& Solanki 1988; Vögler et al. 2005; Vögler 2005; Nordlund et al. 2009; Sánchez Almeida \& Martínez González 2011; Criscuoli 2013). Local dynamo action can create opposite polarity field on small scales $(\sim$ few $\mathrm{km}$ ) that are difficult to observe. We have analyzed their effect on elemental abundance estimates. Specifically, we compared two field morphologies, a small scale magnetic field of zero net vertical flux, represented by our LD atmosphere $\left(\left\langle B_{z}\right\rangle=\right.$ $0 \mathrm{G})$ and an atmosphere with vertical mean field (MF) of $80 \mathrm{G}$ $\left(\left\langle B_{z}\right\rangle=80 \mathrm{G}\right)$.

We find that small scale unresolved magnetic field in the LD case has a small ( $\sim$ few percent) effect on the equivalent 
widths of our spectral line sample and hence induces small deviations in derived abundances of both, $\mathrm{O}$ I and Fe I. Specifically, we found $\left(\Delta A_{\mathrm{O}}\right)_{\mathrm{LD}}=-0.0001$ and $\left(\Delta A_{\mathrm{Fe}}\right)_{\mathrm{LD}}=+0.0044$, for oxygen and iron, respectively, from an unweighted mean of visible lines employed. These values correspond to a few percent or less change in abundance. A vertical imposed mean field has larger impact on equivalent widths and abundance determinations. The arithmetic mean of $\mathrm{O}_{\mathrm{I}}$ lines for the $\mathrm{MF}$ atmosphere gives adjustments of $\left(\Delta A_{\mathrm{O}}\right)_{\mathrm{MF}}=+0.0011$ and the largest changes observed are for iron in the MF simulation with $\left(\Delta A_{\mathrm{Fe}}\right)_{\mathrm{MF}}=+0.0647$. Small scale, randomly oriented field, does not influence abundance estimates significantly, but strong, concentrated, organized field can have an impact. The organization of the field is important.

Abundance estimates are affected by magnetic fields in two ways. Indirectly via a change in the opacity and temperature stratification which acts to decrease the equivalent widths of lines, and directly by Zeeman broadening. These effects on iron lines have been previously studied by Fabbian et al. (2010, 2012). We looked at both oxygen and iron lines, and investigated the relative importance of these two effects in different magnetic field morphologies. A summary of conclusions follows:

Relatively strong $(\|\mathbf{B}\| \sim 1 \mathrm{kG})$ and concentrated vertical magnetic fields modify the opacity and atmospheric thermodynamic stratification, yielding smaller equivalent widths. The MF atmosphere displayed sizable thermodynamic differences from the HD (see Figure 3), and these were most important to the abundance corrections in that case. By contrast, the LD atmosphere showed a more random field configuration that did not alter the atmospheric thermodynamic structure as dramatically.

The net Zeeman effect is larger for solutions with large magnetic field coverage and tends to increase equivalent widths. The LD atmosphere had large areal coverage of moderate field strengths ( $25 \mathrm{G}<\|\mathbf{B}\|<1 \mathrm{kG}$ ) and consequently exhibited abundance corrections influenced by Zeeman broadening. In the LD case the increased Zeeman broadening and reduced indirect thermal effect nearly cancel, leading to smaller abundance corrections of varying sign depending on the magnetic sensitivity of the line.

We conclude that realistic amounts (Rempel 2014) of small scale magnetic field has limited influence on abundance determinations of iron and oxygen. The corrections implied are larger when kilo-Gauss flux concentrations are present, and these form more readily in our MF simulation than our LD simulation, but in either case the implied corrections are very small compared to the changes necessitated by the 1D to 3D modeling transition.

Small scale magnetic fields do not explain the still large discrepancy between spectroscopic abundances and ones consistent with helioseismology.

C.S.M. would like to thank Tom Ayres for his valuable insight and assistance during the project. C.S.M. was supported by
NASA awards NNX13AF55G and NNX13AL35H to the University of Colorado at Boulder. M.R. and M.P.R. were supported in part by NASA award NNX12AB35G. Computing resources included those of the NCAR Wyoming Supercomputing Center via project NHAO0002 on Yellowstone, the NASA Advanced Supercomputing division under project s0925 on Pleiades, and the Janus supercomputer supported by the National Science Foundation (award number CNS-0821794) and the University of Colorado as a joint effort between the University of Colorado and the National Center for Atmospheric Research. NCAR is sponsored by the National Science Foundation.

\section{REFERENCES}

Allende Prieto, C., Asplund, M., \& Fabiani Bendicho, P. 2004, A\&A, 423, 1109

Allende Prieto, C., Lambert, D. L., \& Asplund, M. 2001, ApJL, 556, L63

Asplund, M., Grevesse, N., \& Sauval, A. J. 2005, in ASP Conf. Ser. 336, Cosmic Abundances as Records of Stellar Evolution and Nucleosynthesis, ed. T. G. Barnes \& F. N. Bash, III (San Francisco, CA: ASP), 25

Asplund, M., Grevesse, N., Sauval, A. J., Allende Prieto, C., \& Kiselman, D. 2004, A\&A, 417, 751

Asplund, M., Grevesse, N., Sauval, A. J., \& Scott, P. 2009, ARA\&A, 47,481

Ayres, T. R. 2008, ApJ, 686, 731

Borrero, J. M. 2008, ApJ, 673, 470

Christensen-Dalsgaard, J., di Mauro, M. P., Houdek, G., \& Pijpers, F. 2009, A\&A, 494, 205

Criscuoli, S. 2013, ApJ, 778, 27

Criscuoli, S., Ermolli, I., Uitenbroek, H., \& Giorgi, F. 2013, ApJ, 763, 144

Criscuoli, S., \& Uitenbroek, H. 2014, ApJ, 788, 151

Fabbian, D., Asplund, M., Barklem, P. S., Carlsson, M., \& Kiselman, D. 2009, A\&A, 500, 1221

Fabbian, D., Khomenko, E., Moreno-Insertis, F., \& Nordlund, Å. 2010, ApJ, 724,1536

Fabbian, D., Moreno-Insertis, F., Khomenko, E., \& Nordlund, Å. 2012, A\&A, 548, A 35

Gray, D. 2005, The Observation and Analysis of Stellar Photospheres (Cambridge: Cambridge Univ. Press)

Grevesse, N., Asplund, M., \& Sauval, A. J. 2007, SSRv, 130, 105

Grevesse, N., \& Sauval, A. J. 1998, SSRv, 85, 161

Khomenko, E., \& Collados, M. 2007, ApJ, 659, 1726

Kiselman, D. 1991, A\&A, 245, L9

Martínez Pillet, V. 2013, SSRv, 178, 141

Meyer, D. M., Jura, M., \& Cardelli, J. A. 1998, ApJ, 493, 222

Nissen, P. E., Primas, F., Asplund, M., \& Lambert, D. L. 2002, A\&A, 390,235

Nordlund, A., Stein, R. F., \& Asplund, M. 2009, LRSP, 6, 2

Pereira, T. M. D., Asplund, M., \& Kiselman, D. 2009, A\&A, 508, 1403

Rempel, M. 2014, ApJ, 789, 132

Rempel, M., Schüssler, M., \& Knölker, M. 2009, ApJ, 691, 640

Sánchez Almeida, J., \& Martínez González, M. 2011, in ASP Conf. Ser. 437, Solar Polarization 6, ed. J. R. Kuhn, D. M. Harrington, H. Lin, S. V. Berdyugina, J. Trujillo-Bueno, S. L. Keil, \& T. Rimmele (San Francisco, CA: ASP), 451

Schuessler, M., \& Solanki, S. K. 1988, A\&A, 192, 338

Uitenbroek, H. 2001, ApJ, 557, 389

Uitenbroek, H., \& Criscuoli, S. 2011, ApJ, 736, 69

Vögler, A. 2005, MmSAI, 76, 842

Vögler, A., Shelyag, S., Schüssler, M., et al. 2005, A\&A, 429, 335 